\title{
V680 Mon - a young mercury-manganese star in an eclipsing heartbeat system
}

\author{
Ernst Paunzen ${ }^{1 \star}$ Stefan Hümmerich, ${ }^{2,3}$ Miroslav Fedurco, ${ }^{4}$ Klaus Bernhard, $, 2,3$ \\ Richard Komžík, ${ }^{5}$ Martin Vaňko ${ }^{5}$ \\ ${ }^{1}$ Department of Theoretical Physics and Astrophysics, Masaryk University, Kotlářská 2, 61137 Brno, Czech Republic \\ ${ }^{2}$ Bundesdeutsche Arbeitsgemeinschaft für Veränderliche Sterne e.V. (BAV), Berlin, Germany \\ ${ }^{3}$ American Association of Variable Star Observers (AAVSO), Cambridge, USA \\ ${ }^{4}$ Institute of Physics, Faculty of Science, P. J. Šafárik University, Košice, Slovak Republic \\ ${ }^{5}$ Astronomical Institute of the Slovak Academy of Sciences, 05960 Tatranská Lomnica, Slovak Republic
}

Accepted XXX. Received YYY; in original form ZZZ

\begin{abstract}
Chemically peculiar stars in eclipsing binary systems are rare objects that allow the derivation of fundamental stellar parameters and important information on evolutionary status and the origin of the observed chemical peculiarities. Here we present an investigation of the known eclipsing binary system BD+09 $1467=$ V680 Mon. Using spectra from the Large Sky Area Multi-Object Fiber Spectroscopic Telescope (LAMOST) and own observations, we identify the primary component of the system as a mercury-manganese $(\mathrm{HgMn} / \mathrm{CP} 3)$ star (spectral type kB9 hB8 HeB9 V HgMn). Furthermore, photometric time series data from the Transiting Exoplanet Survey Satellite (TESS) indicate that the system is a 'heartbeat star', a rare class of eccentric binary stars with short-period orbits that exhibit a characteristic signature near the time of periastron in their light curves due to the tidal distortion of the components. Using all available photometric observations, we present an updated ephemeris and binary system parameters as derived from a modelling of the system with the ELISa code, which indicate that the secondary star has an effective temperature of $T_{\text {eff }}=8300_{-200}^{+200}$ (spectral type $\sim \mathrm{A} 4$ ). V680 Mon is only the fifth known eclipsing CP3 star, and the first one in a heartbeat binary. Furthermore, our results indicate that the star is located on the zero-age main sequence and a possible member of the open cluster NGC 2264. As such, it lends itself perfectly for detailed studies and may turn out to be a keystone in the understanding of the development of CP3 star peculiarities.
\end{abstract}

Key words: stars: chemically peculiar — stars: binaries: eclipsing — stars: fundamental parameters

\section{INTRODUCTION}

Among the zoo of chemically peculiar (CP) stars, the mercurymanganese $(\mathrm{HgMn} / \mathrm{CP} 3)$ stars form a rather homogeneous group. They are traditionally identified by the presence of strong $\mathrm{Hg}$ II and $\mathrm{Mn}$ II lines in optical spectra and occupy the rather restricted spectral-type range between B6 and A0 (Preston 1974; Smith 1996; Chojnowski et al. 2020; Paunzen et al. 2021). In addition to strong atmospheric overabundances of $\mathrm{Hg}$ and $\mathrm{Mn}$ (up to 6 and 3 dex over Solar, respectively; e.g. White et al. 1976, Smith 1996, Ghazaryan \& Alecian 2016), CP3 stars exhibit numerous other peculiarities, in particular a general overabundance of heavy elements. Generally, the strength of the overabundances increases with atomic number (Castelli \& Hubrig 2004; Ghazaryan \& Alecian 2016). Detailed

^ E-mail: epaunzen@physics.muni.cz information on the chemical composition of CP3 stars has for example been provided by Castelli \& Hubrig (2004) and Ghazaryan \& Alecian (2016).

CP3 stars are slow rotators (Mathys 2004) and have a high rate of multiplicity. Multiplicity frequencies have been estimated at more than $50 \%$ (Smith 1996), with values up to $67 \%$ (Hubrig \& Mathys 1995) and $91 \%$ (Schöller et al. 2010). CP3 stars do not show the strong magnetic fields that are observed in the Ap/CP2 and the He-peculiar stars (which are lumped together under the label 'magnetic CP stars') and are generally listed with the nonmagnetic CP stars. However, several recent studies announced the presence of weak or tangled fields (Hubrig et al. 2010, 2012) and this has remained a controversial issue (Kochukhov et al. 2013; Hubrig et al. 2020). CP3 stars show an inhomogeneous surface element distribution ('chemical spots') with obvious signs of secular evolution (e.g. Hubrig \& Mathys 1995, Adelman et al. 2002, 
Hubrig et al. 2006, Kochukhov et al. 2007, Briquet et al. 2010, Korhonen et al. 2013).

CP3 stars are relatively rare objects. However, recent progress has led to a substantial extension of the number of known CP3 stars (Chojnowski et al. 2020; Paunzen et al. 2021). At the time of this writing, more than 550 Galactic CP3 stars have been registered. Furthermore, with the advent of space photometry, an increasing number of $\mathrm{CP} 3$ stars has been found to be photometric variables (Alecian et al. 2009; Balona et al. 2011; Morel et al. 2014; Paunzen et al. 2013; Strassmeier et al. 2017; White et al. 2017; Hümmerich et al. 2018). Generally, current studies favoured rotational over pulsational modulation as the cause of the observed variability in the investigated stars (Hümmerich et al. 2018, and the discussion therein).

Given their high multiplicity rate, the presence of a CP3 star component in a number of eclipsing binary systems would be expected. These objects, however, are exceedingly rare: the present authors are only aware of four eclipsing binaries containing a CP3 star component, viz. HD 34364 = AR Aur (Hubrig et al. 2006; Folsom et al. 2010), HD 161701 = HR 6620 (González et al. 2014), TYC 455-791-1 = HSS 348 (Strassmeier et al. 2017), and HD $10260=$ V772 Cas (Kochukhov et al. 2021).

As binaries - in particular eclipsing ones - allow the derivation of fundamental stellar parameters like mass and radius, and only very few CP stars have direct determinations of these parameters (North \& Debernardi 2004), the discovery of binary systems with CP star components is important. Furthermore, such systems may help to understand, and put constraints on, the processes responsible for the formation of the observed chemical peculiarities and the time scales involved.

Here we report on the discovery of another eclipsing binary system containing a CP3 star component, viz. BD+09 1467= V680 Mon, which is well suited to follow-up studies dealing with the solution of the system and the determination of exact stellar parameters for both components. We show that V680 Mon is a 'heartbeat' star, a rare class of eccentric binary stars with short-period orbits $\left(1 \mathrm{~d} \underset{\sim}{<} P_{o r b} \lesssim 1 \mathrm{yr}\right)$ that exhibit a characteristic signature near the time of periastron in their light curves whose shape is reminiscent of an electrocardiogram diagram (hence the name).

Section 2 provides information on our target star, its astrophysical parameters and location in the sky, and the observations. We present our results in Section 3 and discuss them in Section 4.

\section{TARGET STAR, OBSERVATIONS AND DATA ANALYSIS}

\subsection{Target star}

V680 Mon = BD+09 $1467=$ HD 267564 (spectral type B8, Cannon \& Pickering 1993; $V=10.13 \mathrm{mag}$, ESA 1997; $G=9.98 \mathrm{mag}$, Gaia Collaboration et al. 2018) was identified as a variable star by Parenago (1946), who listed it under the preliminary designation of SVS 1025 Monocerotis and suggested it to be an eclipsing binary with a range of $9.5-10.1 \mathrm{mag}(\mathrm{pg})$. No period could be derived from the available observations. The star was included as NSV 3233 into the New Catalogue of Suspected Variable Stars (Kukarkin \& Kholopov 1982) and later entered the General Catalogue of Variable Stars as V680 Mon (Kholopov et al. 1987; Samus et al. 2017).

V680 Mon has been little studied, and discrepant information is found in the literature. From an analysis of 85 photographic

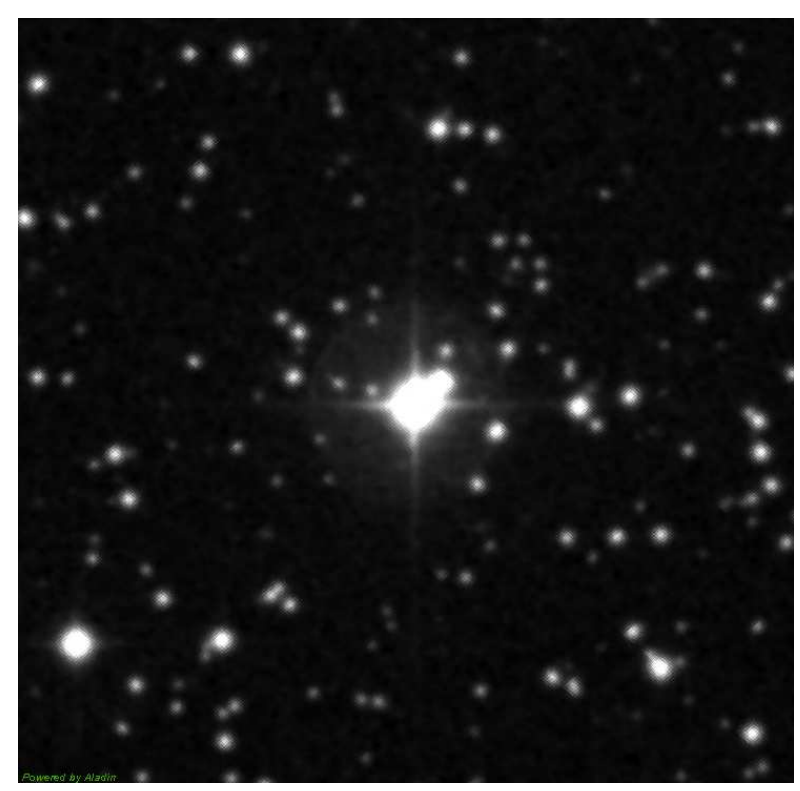

Figure 1. Sky region of V680 Mon on DSS2 blue plates, accessed via the ALADIN sky atlas (Bonnarel et al. 2000; Boch \& Fernique 2014). The field of view is $4.289^{\prime} \times 4.108^{\prime}$. North is at the top, east is at the left. The image is centered on V680 Mon. The bright star to the north-west that appears partially blended with V680 Mon is 2MASS J06593015+0919070.

plates, Berthold (1983) ${ }^{1}$ proposed V680 Mon to be an RR Lyrae star and derived first (but, in hindsight, incorrect) elements. Presumably based on this information, V680 Mon was included in the RR Lyrae star catalogues of Mennessier \& Colomé (2002) and Maintz (2005). However, on the basis of ASAS-3 and NSVS observations, Otero et al. (2006) identified V680 Mon as an eclipsing binary star, in accordance with the initial proposition of Parenago (1946). The authors derived a period of $P=8.5381 \mathrm{~d}$ and a variability range of $9.93-10.31 \mathrm{mag}(V)$. The system was found to be eccentric, with the secondary minimum occurring at phase $\varphi=0.865$. In consequence, V680 Mon entered the catalogue of eclipsing binary stars with eccentric orbits by Bulut \& Demircan (2007) and new observations of minima were procured by Brat et al. (2009) and Hübscher (2011).

Despite these results, and the correct identification of the star as an eclipsing binary in the International Variable Star Index (VSX; Watson 2006) of the American Association of Variable Star Observers (AAVSO), the star has been listed as an RR Lyrae variable in the SIMBAD database (Wenger et al. 2000) until recently, which is probably the reason why it was included into the samples of the RR-Lyrae-star-based studies of Gavrilchenko et al. (2014) and Gaia Collaboration et al. (2017). On the initiative of the present authors, V680 Mon is now correctly identified in the SIMBAD database as an eclipsing binary star.

${ }^{1}$ https://www. sternwarte-hartha.de/wp-content/uploads/ 
Table 1. Basic stellar parameters of V680 Mon (2MASS J06593071+0918596) and its close companion 2MASS J06593015+0919070.

\begin{tabular}{lllllllllllll}
\hline \hline ID & Gaia DR2 & $\alpha$ & $\delta$ & $\pi$ & e $\pi$ & $G$ mag & e $_{-} G$ mag & $(B P-R P)_{0}$ & $\mathrm{e}_{-}(B P-R P)_{0}$ & $\mathrm{MV}_{0}$ & $\mathrm{e}_{-} \mathrm{MV} \mathrm{V}_{0}$ \\
\hline $\mathrm{J} 06593071+0918596$ & 3157882748862195072 & 104.8779802 & 9.3165762 & 1.594 & 0.146 & 9.9803 & 0.0011 & 0.000 & 0.004 & 1.09 & 0.16 \\
$\mathrm{~J} 06593015+0919070$ & 3157882744564424704 & 104.8756241 & 9.3186280 & 0.370 & 0.018 & 12.5163 & 0.0002 & 1.146 & 0.002 & 0.35 & 0.09 \\
\hline \hline
\end{tabular}

\subsection{Sky region}

V680 Mon is situated in an area relatively devoid of bright stars, roughly in the midst of an imaginary triangle with Alhena ( $\gamma \mathrm{Gem})$, Procyon ( $\alpha \mathrm{CMi}$ ) and the Rosette Nebula (NGC 2244) as its vortices. However, situated at a distance of $12^{\prime \prime}$ from our target star, there is the relatively bright star 2MASS J06593015+0919070 = GAIA DR2 $3157882744564424704(G=12.52 \mathrm{mag}$, Gaia Collaboration et al. 2018). Both stars appear as a close double in DSS2 images (Fig. 1). They will also be blended in the spectroscopic and photometric data that form the backbone of this investigation; hence, a more detailed investigation into this matter is necessary.

Parameters of both stars are given in Table 1. Bailer-Jones et al. (2020) list distances of, respectively, 619 pc (581-672 pc) and $2556 \mathrm{pc}(2438-2649 \mathrm{pc})$ for V680 Mon and 2MASS J06593015+0919070 based on the Gaia Early Data Release 3 (EDR3, Gaia Collaboration et al. 2020). The two stars are not physically connected to each other. Considering its colour and luminosity, 2MASS J06593015+0919070 is obviously a late G- or early K-type giant. It is about 10 times $(\sim 2.5 \mathrm{mag})$ fainter than V680 Mon, and we expect no significant contamination of the here employed spectra, which do not show any traces of the signature of a late G- or early K-type giant. Furthermore, we can rule out that the eclipses observed in the combined light curve of both stars originate in 2MASS J06593015+0919070. Even its complete disappearance would not result in a $25 \%$ reduction in brightness, as is observed during primary eclipse of the system. We are therefore confident that no blending issues affect our main results.

\subsection{Observations}

The spectra employed in this study were extracted from the archive of the Large Sky Area Multi-Object Fiber Spectroscopic Telescope $(\text { LAMOST })^{2}$, which is located at Xinglong Observatory in Beijing (China), and procured at Stará Lesná Observatory (SLO) and Skalnaté Pleso Observatory (SPO) in the High Tatras (Slovak Republic).

The LAMOST low-resolution spectrum boasts a resolution of $\mathrm{R} \sim 1800$ and covers the wavelength range from 3700 to $9000 \AA$. More information on the LAMOST survey is provided in Zhao et al. (2012) and Cui et al. (2012). The spectra taken at SLO have a resolution between $R=11000$ and $R=12000$ and cover the spectral range from 4150 to $7600 \AA$, while the spectra procured at SPO have a resolution of $R=38000$ and cover the interval from 4250 to $7375 \AA$. More information on the instrumentation of the SLO and SPO observatories and the reduction process can be found in Pribulla et al. (2015).

The photometric observations used in this study were procured by NASA's Transiting Exoplanet Survey Satellite (TESS), which provides ultra-precise photometry in a single passband (600$1000 \mathrm{~nm}$ ) taken at a cadence of $2 \mathrm{~min}$. More information on the
TESS spacecraft and data products can be found in Ricker et al. (2014, 2015); Campante et al. (2016).

\section{RESULTS}

\subsection{Spectral classification}

The spectral peculiarities of V680 Mon were discovered during our semi-automated search for new CP3 stars in the spectra from LAMOST DR4 (Paunzen et al. 2021). Because it was recognised as an eclipsing binary and further studies were deemed necessary, V680 Mon was not included into the sample of Paunzen et al. (2021). More information on our search for CP stars with Richard O. Gray's MKCLASS code, a program that classifies spectra by emulating the workflow of a human classifier (Gray \& Corbally 2014), can be found in Hümmerich et al. (2020) and Paunzen et al. (2021).

Only one LAMOST spectrum is available for V680 Mon = LAMOST J065930.88+091859.6, which was accessed via the DR4 VizieR online catalogue ${ }^{3}$ (Luo et al. 2018). The spectrum was obtained on 28 December 2015 (MJD 57384; observation median UTC 17:38:00; $g$-band S/N: 294), that is, at an orbital phase of $\varphi=0.705$. It was therefore obtained during maximum light and is dominated by light from the primary component of the V680 Mon system. The spectrum is illustrated in Fig. 2, together with the synthetic spectrum of a B8 V star and the LAMOST DR4 spectrum of an $\mathrm{HgMn}$ star from the list of Paunzen et al. (2021).

Following the workflow outlined in Paunzen et al. (2021), V680 Mon is given a final classification of B9 III-IV $\mathrm{HgMn}$ by the employed specialised version of the MKCLASS code. The Ca II $\mathrm{K}$ line strength and the relatively weak $\mathrm{He}$ II lines indeed suggest a spectral type of B9; the hydrogen line profile, however, is best matched by that of a B8 V standard. In general, the hydrogen-line profile is the most reliable indicator of the effective temperature in a CP star (Gray \& Corbally 2009). Furthermore, CP3 stars have been shown to exhibit a large spread of He abundances, with most (usually the hotter) CP3 stars being He deficient (Smith 1996; Ghazaryan \& Alecian 2016). We therefore prefer a temperature type of B8 V. While CP3 stars also show a large dispersion of Ca abundances (up to 3 dex; Ghazaryan \& Alecian 2016), we suspect that there is an interstellar contribution to the strong $\mathrm{Ca}$ II $\mathrm{K}$ line in the available spectrum.

The CP3 star characteristics are clearly present in the spectrum of V680 Mon. The Hg II $3984 \AA$ line appears merely as a 'bump' in the red wing of $\mathrm{H} \epsilon$, as is commonly the case at this low resolution (cf. e.g. the CP3 star spectra shown in Paunzen et al. 2021). The Mn II features at $4136 \AA$ and, in particular, $4206 \AA$ and $4152 / 9 \AA$ are well developed (Fig. 2). In summary, following the refined classification system of Garrison \& Gray (1994), we arrive at a final classification of kB9 hB8 HeB9 V HgMn. 


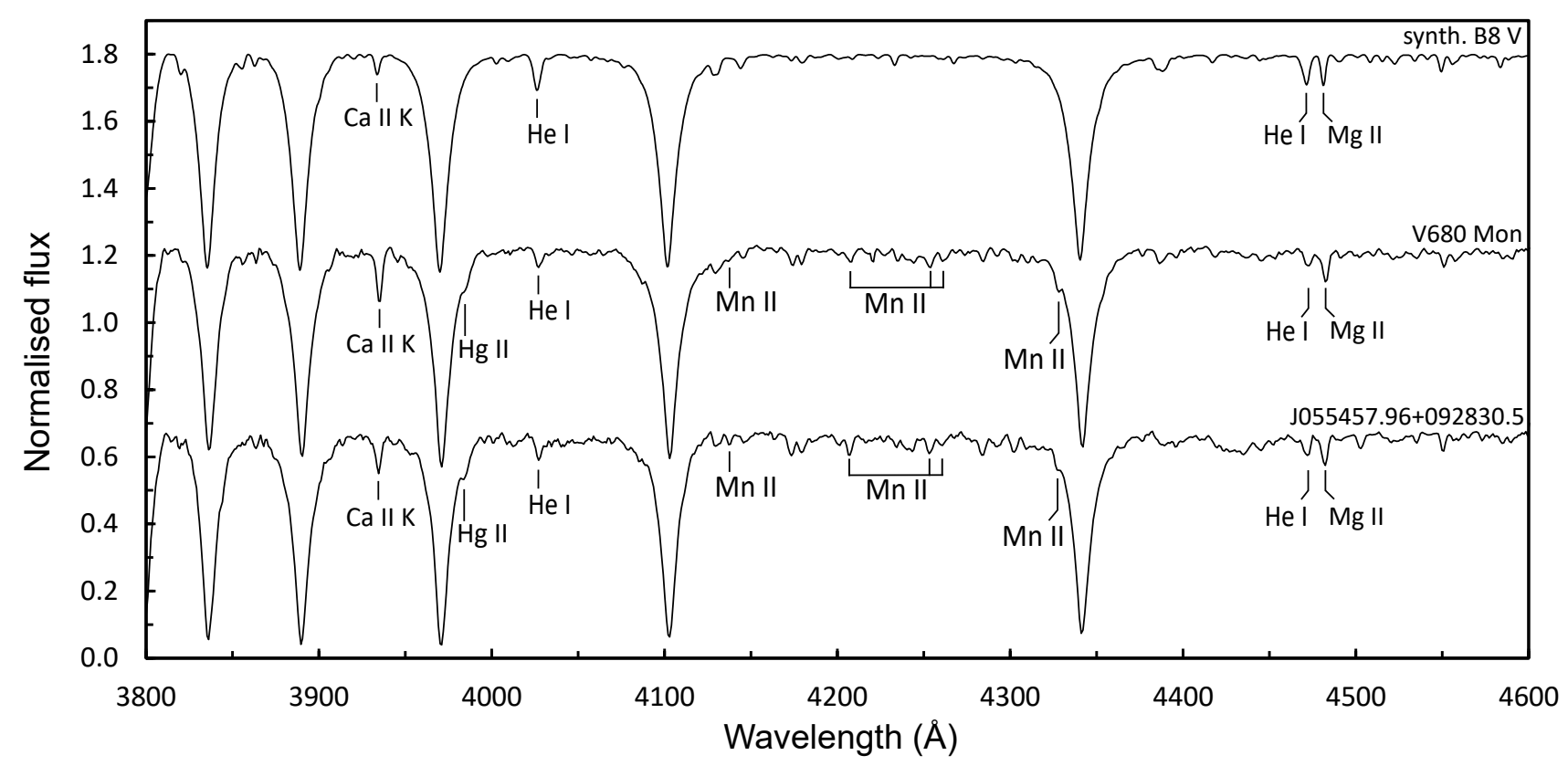

Figure 2. Comparison of the blue-violet region of (a) a synthetic spectrum corresponding to spectral type B8 V $\left(T_{\text {eff }}=12500 \mathrm{~K} ; \log g=4.0 ;[\mathrm{M} / \mathrm{H}]=0.0\right.$; $\xi=2 \mathrm{~km} \mathrm{~s}^{-1}$; smoothed to match the LAMOST resolution), (b) the LAMOST DR4 spectrum of V680 Mon (kB9 hB8 HeB9 V HgMn), and (c) the LAMOST DR4 spectrum of the CP3 star HD 249170 = LAMOST J055457.96+092830.5 (B8 IV HgMn; Paunzen et al. 2021). Some prominent lines of interest are identified.

We analysed the high-resolution SLO and SPO spectra obtained at five different orbital phases and found no traces of the secondary component. V680 Mon, therefore, is a single-line spectroscopic binary (SB1) system. In addition to the classical classification criteria discussed above, we measured the equivalent widths of the $\mathrm{Mn}$ I lines at 4462.031, 4762.367, 4765.846, 4766.418, $4783.427,4823.524$, and $6021.790 \AA$, all of which exhibit values between 15 and $20 \mathrm{~m} \AA$. This is well in line with the results obtained for the HgMn star HD $175640([\mathrm{Mn}]=+2.45$ dex and $[\mathrm{Hg}]=+4.72$ dex as compared to the Sun; Castelli \& Hubrig 2004), which has an identical effective temperature as our target star. We also investigated the weak $\mathrm{Hg}_{\text {I }} 5460.731 \AA$ line, which yields an equivalent width of $3 \mathrm{~m} \AA$. While this is at the detection limit of our set of spectra, its presence clearly indicates a significant overabundance of $\mathrm{Hg}$; for solar metallicity, the equivalent width of this line is well below $1 \mathrm{~m} \AA$. In summary, our analysis of the SLO and SPO spectra corroborates the results from the blue-violet spectral region and confirms that the primary component of V680 Mon is a CP3 star.

\subsection{Photometric variability}

On the basis of ASAS-3 and NSVS observations, Otero et al. (2006) identified V680 Mon as an eclipsing binary star (cf. Section 2.1). They derived a period of $P=8.5381 \mathrm{~d}$ (epoch of primary miminum: HJD 2452990.717), a total range of light variability of $9.93-10.31 \mathrm{mag}(V)$ and found the system to be eccentric, with the secondary minimum occurring at phase $\varphi=0.865$. With these data, the star was included into the VSX.

V680 Mon was observed by TESS during orbits 19 and 20 (TESS Observation Sector 6). The corresponding data were accessed via 'eleanor', which is an open-source python frame- work for downloading, analysing, and visualising data from the TESS Full Frame Images (Feinstein et al. 2019). ${ }^{4}$ In the direct vicinity of our target star, there is the relatively bright star 2MASS J06593015+0919070 $(G=12.52 \mathrm{mag})$, which is separated from V680 Mon by a distance of approximately 12" (cf. Section 2.2). Since the TESS pixel size is relatively large (21"), the TESS light curve is a blending of light from both stars. However, as discussed in Section 2.2, the light contribution from 2MASS J06593015+0919070 is negligible and cannot account for the observed eclipses. Apart from its close neighbor, V680 Mon is rather isolated from other bright stars. We tried both the PCA and PSF reductions and found that the PSF-modelled light curve is superior. For the final analysis, PSF modelling using a field of $7 \mathrm{x} 7$ pixels was employed.

The TESS light curve of V680 Mon is illustrated in Fig. 3. Although the covered time-span is short, the ultra-precise TESS data reveal for the first time that V680 Mon is a heartbeat system. A detailed view of the heartbeats near periastron, whose shape is due to the combined effects of tidal distortion, reflection and Doppler beaming (Hambleton et al. 2013; Fuller 2017; Hambleton et al. 2018), is presented in Figure 4. The presence of chemical peculiarities in the component of a binary of 'heartbeat configurating' is an interesting find that is further discussed in Section 4.

To derive an updated ephemeris, we combined the available photometric time-series data from TESS, the All Sky Automated Survey (ASAS-3; Pojmanski 2002) and the Kamogata/Kiso/Kyoto

\footnotetext{
${ }^{4}$ https://adina.feinste.in/eleanor/
} 


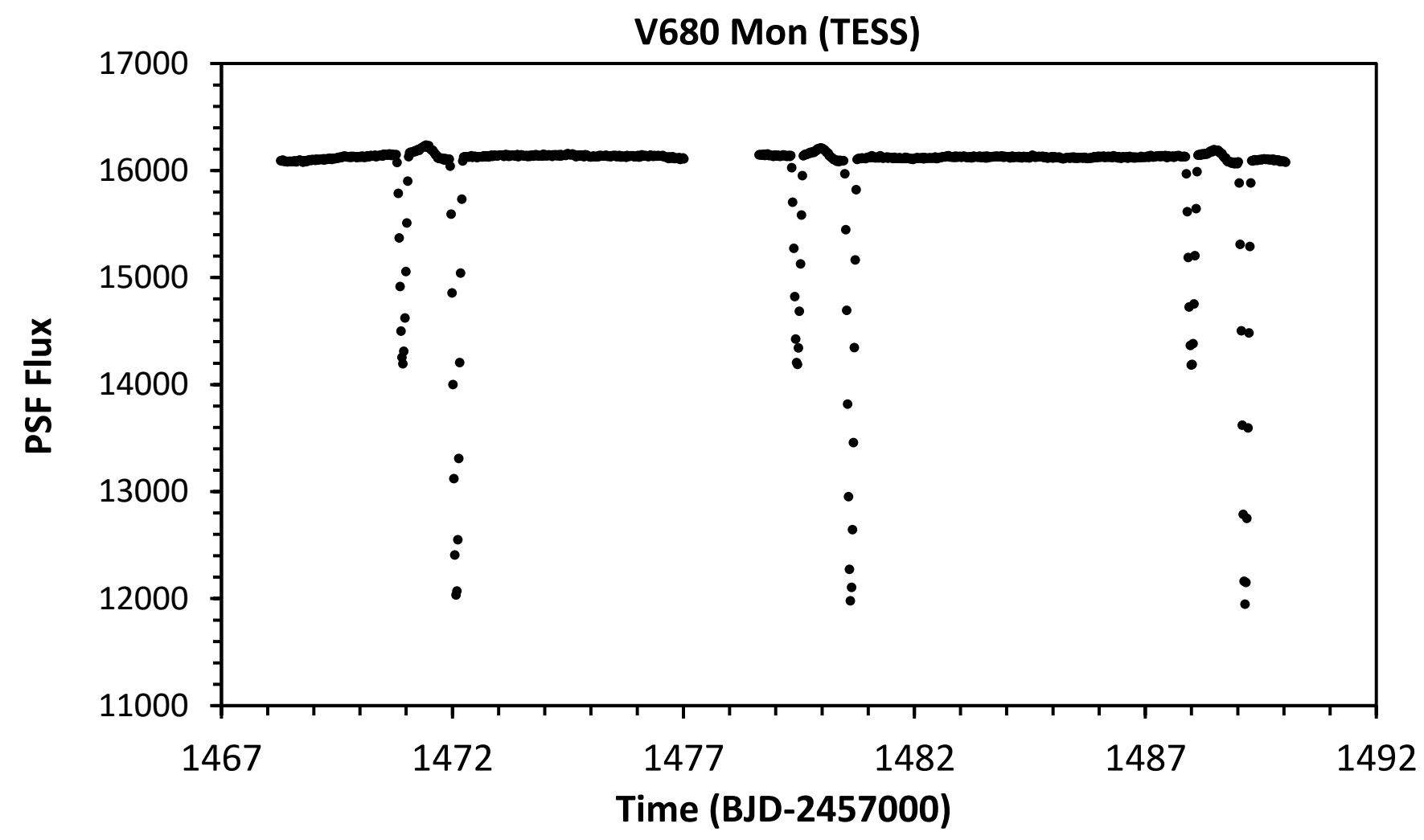

Figure 3. TESS light curve of V680 Mon, accessed via 'eleanor' and based on PSF flux.

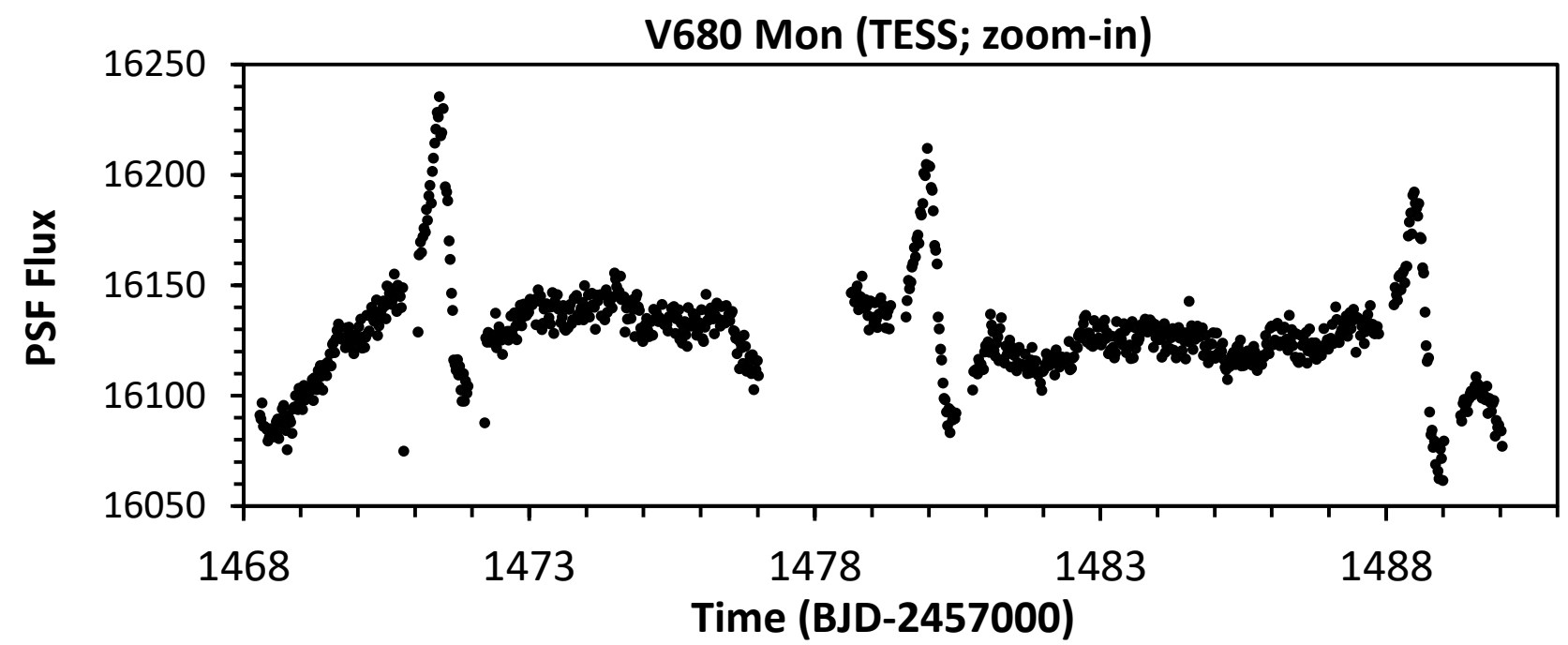

Figure 4. TESS light curve of V680 Mon, accessed via 'eleanor' and based on PSF flux, presenting a detailed view of the 'heartbeats'.

wide-field survey (KWS; Maehara 2014) and derived the elements presented in Eqs. 1 and 2.

Min $I=H J D 2458472.088(1)+8.53797(2) E$

Min II = HJD 2458470.929(2) + 8.53797(2) E
3.3 Binary system modelling

Due to the significantly lower quality of the ASAS-3 and KWS photometry, binary system parameters were inferred using only detrended TESS photometry. The light curve was analysed with the 


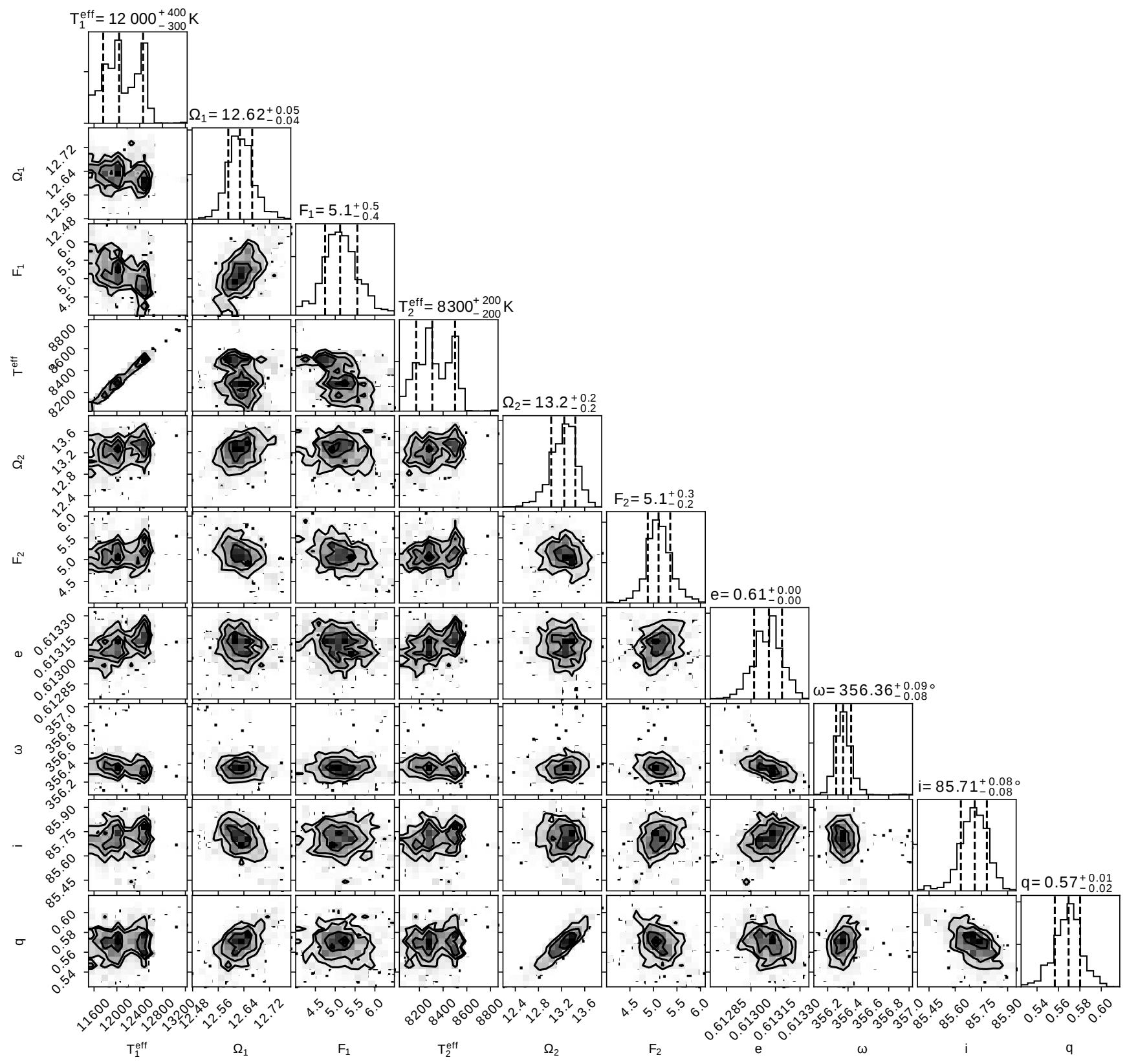

Figure 5. Posterior distribution of the samples generated by the Markov Chain Monte Carlo (MCMC) algorithm.

Python package ELISa ${ }^{5}$, which contains tools for modelling light curves of close eclipsing binaries utilising Roche geometry and methods for solving an inverse problem.

As a first step, the Least-Squares Trust Region Reflective Algorithm ('Least Squares' hereafter) was used to search for a local minimum around a manually-selected starting point based on the general shape of the light curve. Initial runs were performed with seven free parameters: photometric mass ratio $q$; inclination $i$; eccentricity $e$; argument of periastron $\omega$; surface potentials $\Omega_{1}, \Omega_{2}$; and effective temperature of the secondary component $T_{2}^{e f f}$. The effective temperature of the primary component $T_{1}^{e f f}$ was fixed to

\footnotetext{
5 https://github.com/mikecokina/elisa
}

$12500 \mathrm{~K}$, as inferred from the spectral classification (cf. Section 3.1). Albedos $A_{1}, A_{2}$ and gravity darkening factors $\beta_{1}, \beta_{2}$ were set to 1.0 since both components were expected to have radiative envelopes due to their effective temperatures being well above $7000 \mathrm{~K}$ (Zeipel 1924). We adopted a square-root law for limb darkening. The corresponding coefficients were interpolated from the precalculated tables of Claret (2017), and atmospheric models from Castelli \& Kurucz (2004) were used for the calculation of the integrated passband flux. Finally, the synchronicity factors $F_{1}, F_{2}$ were set to assume synchronous rotation of the components at periastron (Hut 1981).

After the initial solution was found, the effective temperature of the primary component $T_{1}^{\text {eff }}$, synchronicity parameters $F$, gravity darkening factors $\beta$, and albedos $A$ were set variable to allow 
Table 2. Parameters of the V680 Mon system as obtained from the LeastSquares Trust Region Reflective Algorithm ('Least Squares') and subsequent error estimation using the Markov Chain Monte Carlo (MCMC) sampler. The components' radii are given in semi-major axis units (SMA).

\begin{tabular}{|c|c|c|c|}
\hline Parameter & \multicolumn{2}{|c|}{ Value } & Status \\
\hline \multicolumn{4}{|l|}{ System } \\
\hline$q$ & & Variable \\
\hline$i\left[^{\circ}\right]$ & \multicolumn{2}{|c|}{$\begin{array}{c}-0.02 \\
85.71_{-0.08}^{+0.08}\end{array}$} & Variable \\
\hline$e$ & \multicolumn{2}{|c|}{$0.6131_{-1 \times 10^{-4}}^{-0.08}$} & Variable \\
\hline$\omega\left[^{\circ}\right]$ & \multicolumn{2}{|c|}{$\begin{array}{c}356.36_{-0.08}^{+0.09} \\
30^{-4}\end{array}$} & Variable \\
\hline$P[d]$ & \multicolumn{2}{|c|}{8.53797} & Fixed \\
\hline$T_{0}[d]$ & \multicolumn{2}{|c|}{2458472.088} & Fixed \\
\hline Component & primary & secondary & \\
\hline$\Omega$ & $12.62_{-0.04}^{+0.04}$ & $13.2_{-0.2}^{+0.2}$ & Variable \\
\hline$F$ & $5.1_{-04}^{+0.5} 04$ & $5.1_{-0.0}^{+0.2}$ & Variable \\
\hline$r_{e q}$ & $0.0836_{-0.0002}^{+0.0002}$ & $0.0474_{-0.004}^{+0.005}$ & Derived \\
\hline \multicolumn{4}{|c|}{ Atmospheric parameters } \\
\hline$T^{e f f} /[K]$ & $12000_{-300}^{+400}$ & $8300_{-200}^{+200}$ & Variable \\
\hline$\beta$ & $0.86_{-0.05}^{+0.04}$ & $0.89_{-0.04}^{+0.03}$ & Variable \\
\hline A & $0.73_{-0.07}^{-0.05}$ & $0.64_{-0.04}^{+0.04}$ & Variable \\
\hline$M / H$ & 0.0 & $0.0^{-0.04}$ & Fixed \\
\hline \multicolumn{4}{|c|}{ Radii at periastron } \\
\hline$r_{\text {pole }}$ & $0.0827_{-0.0003}^{+0.0003}$ & $0.0472_{-0.0004}^{+0.0005}$ & Derived \\
\hline$r_{\text {back }}$ & $0.0844_{-0.0002}^{+0.0002}$ & $0.0476_{-0.0004}^{+0.0004}$ & Derived \\
\hline$r_{\text {side }}$ & $0.0837_{-0.0002}^{+0.0002}$ & $0.0474_{-0.0004}^{+0.0005}$ & Derived \\
\hline$r_{\text {forward }}$ & $0.0846_{-0.0002}^{+0.0002}$ & $0.0476_{-0.0004}^{+0.0005}$ & Derived \\
\hline
\end{tabular}

for the model to relax into the local minimum. $T_{1}^{e f f}$ was allowed to vary $\pm 1000 \mathrm{~K}$. Using the approach described above, the Least Squares algorithm arrived at a solution with a coefficient of determination of $R^{2}=0.9990$.

The vicinity of the obtained solution was sampled using the Markov Chain Monte Carlo algorithm (MCMC) with 200 walkers, 200 steps, and uniform prior sampling. After discarding the thermalisation phase of the chain, the confidence intervals of the parameters were inferred from the posterior distribution displayed in Figure 5 in the form of a corner plot. The resulting system parameters are listed in Table 2 and the corresponding fit is illustrated in Figure 6.

The achieved solution points to an eccentric orbit with eccentricity $e=0.61$ and a relatively short orbital period. The tidal forces during the periastron passage result in a deformation of both components with an amplitude of $\sim 1 \%$ (ratio between forward and equivalent radius). The synchronicity parameters for both components remained within errors around the predicted value of 5.27, which suggests that the rotation of the components is synchronised with the orbital motion during the periastron. Figure 5 shows the double Gaussian distribution for both effective temperatures that indicates two solutions with similar qualities of fit. However, since their spacing is similar to the standard deviations of each peak, we decided to regard them as a single solution. No additional double Gaussian distributions were detected for any other variable parameters. We note that the fitting process based on information obtained in a single passband has a limited capacity to recover information about the effective temperature of the components.

\section{DISCUSSION}

The evolutionary stages of the four known eclipsing binary systems containing a CP3 star component are widely different (González et al. 2014; Kochukhov et al. 2021), ranging from close to the zeroage main sequence (HD 34364 and TYC 455-791-1) to the middle of the main sequence (HD 161701) and close to the terminal-age main sequence (HD 10260).

To evaluate the evolutionary status of V680 Mon, we investigated its position in the Hertzprung-Russell diagram. As first step, the absolute magnitude listed in Table 1 was corrected for the contribution of the secondary component as shown in Fig. 9 of Paunzen et al. (2021). For the given $q$ value (Table 2), this correction amounts to $0.15 \mathrm{mag}$ only. The bolometric correction for CP stars (Netopil et al. 2008) yields a value of -0.559 mag and thus a luminosity of $\log L / L_{\odot}=1.628(64)$. For the calibration of the age, we used the Stellar Isochrone Fitting Tool $^{6}$ and the isochrone grid by Bressan et al. (2012) for solar metallicity.

Our results indicate that V680 Mon is located on the zeroage main sequence with an age between 5 and $6 \mathrm{Myr}$. This result is not dependent on the choice of the isochrone metallicity because the use of overabundant isochrones would result in an even larger luminosity for the same effective temperature. In such grids, our target star would be situated significantly below the zero-age main sequence.

Several CP3 stars belong to open clusters (e.g. Hubrig et al. 2012), which puts further constraints on the age determination. We searched for a possible host cluster within $3 \sigma$ of the position, diameter, proper motion, distance and their errors of the star cluster lists from Dias et al. (2002) and Cantat-Gaudin \& Anders (2020). Because V680 Mon is quite close (about 620 pc from the Sun), we expect these lists to be complete. The closest aggregate is NGC 2264, which is located about 4.6 degrees or $50 \mathrm{pc}$ away. In this young open cluster, star formation is still going on (Nony et al. 2021) and many young stellar objects are present (Buckner et al. 2020). If we accept V680 Mon as a member of NGC 2264, the derived ages are in excellent agreement. Incidentally, another $\mathrm{HgMn}$ star, HD 47553, was reported as a member of NGC 2264 (Pyatkes 1993); unfortunately, besides this reference, no other analysis of this star was found in the literature.

V680 Mon is only the fifth known eclipsing CP3 star, and it is the first one recognised as member of a heartbeat binary. Our results indicate that the V680 Mon system is composed of a CP3 star primary component $\left(T_{e f f}=12000_{-300}^{+400} \mathrm{~K}\right.$; spectral type kB9 hB8 HeB9 V HgMn) and a secondary component of spectral type A4 $\left(T_{e f f}=8300_{-200}^{+200}\right.$; cf. Section 3.3). The unique combination of a very young and relatively bright chemically peculiar star in such a system opens up intriguing possibilities. In particular, theory needs to explain the development of CP3 star features in such a young object and under the conditions (tidally-induced effects) of a heartbeat binary. Our modelling attempts indicate a significant deformation of both components by about one per cent (ratio between forward and equivalent radius) during periastron passage.

In this respect, it is interesting to note that there is a high rate of occurrence of very eccentric short-period binary systems among the CP1 stars (Debernardi 2000), which some studies have proposed as lower-temperature counterparts of the CP3 stars (Adelman et al. 2003). Obviously, the tidally-induced effects do not interfere with the development of CP1 star abundance patterns in these systems. CP2, CP3 and CP4 stars, on the other hand, show a rather dif-

6 https://github.com/Johaney-s/StIFT 


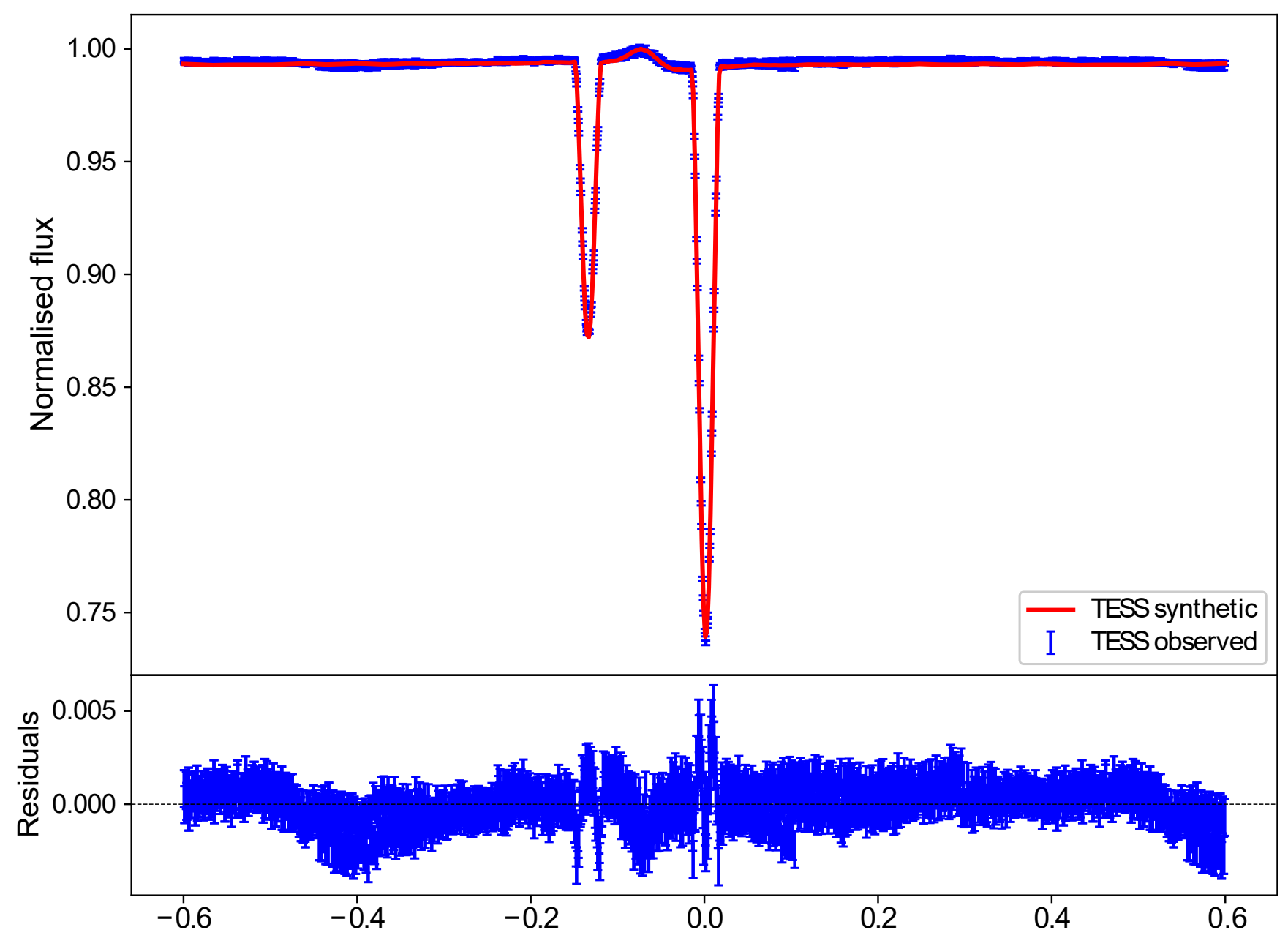

Figure 6. The upper panel illustrates the fit between observed and synthetic flux in the TESS passband based on the best-fit model presented in Table 2. The fitting residuals are shown in the lower panel.

ferent eccentricity versus orbital period distribution with an apparent upper envelope (Carrier et al. 2002). Interestingly, V680 Mon is located well above this proposed upper envelope (cf. Fig. 8 of Carrier et al. 2002).

In summary, V680 Mon lends itself perfectly for detailed follow-up studies and may prove to be a keystone in the understanding of the development of CP3 star peculiarities.

\section{ACKNOWLEDGEMENTS}

We thank the referee, Gautier Mathys, for his comments that helped to improve the paper. We furthermore thank Theodor Pribulla and Johana Supíková for their help in preparing this manuscript and express our gratitude to Iosif I. Romanyuk for providing the original Peremennye Zvezdy paper of P. P. Parenago and Hans Michael Maitzen for his translation of the Russian original. EP acknowledges support by the Erasmus+ programme of the European Union under grant number 2020-1-CZ01-KA203-078200. This work has been supported by the VEGA grants of the Slovak Academy of Sciences No. 2/0031/18 and 2/0004/20. This paper makes use of data from the Guoshoujing Telescope (the Large Sky Area Multi-Object Fiber Spectroscopic Telescope LAMOST), which is a National Major Scientific Project built by the Chinese Academy of Sciences. Funding for the project has been provided by the National Development and Reform Commission. LAMOST is operated and managed by the National Astronomical Observatories, Chinese Academy of Sciences. Furthermore, this paper includes data collected by the TESS mission. Funding for the TESS mission is provided by the NASA's Science Mission Directorate. This work presents results from the European Space Agency (ESA) space mission Gaia. Gaia data are being processed by the Gaia Data Processing and Analysis Consortium (DPAC). Funding for the DPAC is provided by national institutions, in particular the institutions participating in the Gaia MultiLateral Agreement (MLA). The Gaia mission website is https://www.cosmos.esa.int/gaia. The Gaia archive website is https://archives.esac.esa.int/gaia. This research has made use of the WEBDA database, operated at the Department of Theoretical Physics and Astrophysics of the Masaryk University, and the SIMBAD and VizieR databases, operated at CDS, Strasbourg, France. 


\section{DATA AVAILABILITY}

The data underlying this article will be shared on reasonable request to the corresponding author.

\section{REFERENCES}

Adelman S. J., Gulliver A. F., Kochukhov O. P., Ryabchikova T. A., 2002, ApJ, 575, 449

Adelman S. J., Adelman A. S., Pintado O. I., 2003, A\&A, 397, 267

Alecian G., Gebran M., Auvergne M., Richard O., Samadi R., Weiss W. W., Baglin A., 2009, A\&A, 506, 69

Bailer-Jones C. A. L., Rybizki J., Fouesneau M., Demleitner M., Andrae R., 2020, arXiv e-prints, p. arXiv:2012.05220

Balona L. A., et al., 2011, MNRAS, 413, 2403

Berthold T., 1983, Mitteilungen der Bruno-H.-Bürgel-Sternwarte, 18, 1

Boch T., Fernique P., 2014, in Manset N., Forshay P., eds, Astronomical Society of the Pacific Conference Series Vol. 485, Astronomical Data Analysis Software and Systems XXIII. p. 277

Bonnarel F., et al., 2000, A\&AS, 143, 33

Brat L., et al., 2009, Open European Journal on Variable Stars, 107, 1

Bressan A., Marigo P., Girardi L., Salasnich B., Dal Cero C., Rubele S., Nanni A., 2012, MNRAS, 427, 127

Briquet M., Korhonen H., González J. F., Hubrig S., Hackman T., 2010, A\&A, 511, A71

Buckner A. S. M., et al., 2020, A\&A, 636, A80

Bulut I., Demircan O., 2007, MNRAS, 378, 179

Campante T. L., et al., 2016, ApJ, 830, 138

Cannon A. J., Pickering E. C., 1993, VizieR Online Data Catalog, p. III/ $135 \mathrm{~A}$

Cantat-Gaudin T., Anders F., 2020, A\&A, 633, A99

Carrier F., North P., Udry S., Babel J., 2002, A\&A, 394, 151

Castelli F., Hubrig S., 2004, A\&A, 425, 263

Castelli F., Kurucz R. L., 2004, preprint (arXiv: 0405. 08)

Chojnowski S. D., Hubrig S., Hasselquist S., Beaton R. L., Majewski S. R., García-Hernández D. A., DeColibus D., 2020, MNRAS,

Claret A., 2017, A\&A, 600, A30

Cui X.-Q., et al., 2012, Research in Astronomy and Astrophysics, 12, 1197

Debernardi Y., 2000, IAU Symposium, 200, 161

Dias W. S., Alessi B. S., Moitinho A., Lépine J. R. D., 2002, A\&A, 389, 871

ESA ed. 1997, The HIPPARCOS and TYCHO catalogues. Astrometric and photometric star catalogues derived from the ESA HIPPARCOS Space Astrometry Mission ESA Special Publication Vol. 1200

Feinstein A. D., et al., 2019, PASP, 131, 094502

Folsom C. P., Kochukhov O., Wade G. A., Silvester J., Bagnulo S., 2010, MNRAS, 407, 2383

Fuller J., 2017, MNRAS, 472, 1538

Gaia Collaboration et al., 2017, A\&A, 605, A79

Gaia Collaboration et al., 2018, A\&A, 616, A1

Gaia Collaboration Brown A. G. A., Vallenari A., Prusti T., de Bruijne J. H. J., Babusiaux C., Biermann M., 2020, arXiv e-prints, p. arXiv:2012.01533

Garrison R. F., Gray R. O., 1994, AJ, 107, 1556

Gavrilchenko T., Klein C. R., Bloom J. S., Richards J. W., 2014, MNRAS, 441,715

Ghazaryan S., Alecian G., 2016, MNRAS, 460, 1912

González J. F., et al., 2014, A\&A, 561, A63

Gray R. O., Corbally C. J., 2009, Stellar Spectral Classification. Princeton, NJ: Princeton Univ. Press

Gray R. O., Corbally C. J., 2014, The Astronomical Journal, 147, 80

Hambleton K., et al., 2013, in Pavlovski K., Tkachenko A., Torres G., eds, EAS Publications Series Vol. 64, EAS Publications Series. pp 285-294, doi:10.1051/eas/1364039

Hambleton K., et al., 2018, MNRAS, 473, 5165

Hubrig S., Mathys G., 1995, Comments on Astrophysics, 18, 167
Hubrig S., González J. F., Savanov I., Schöller M., Ageorges N., Cowley C. R., Wolff B., 2006, MNRAS, 371, 1953

Hubrig S., et al., 2010, MNRAS, 408, L61

Hubrig S., et al., 2012, A\&A, 547, A90

Hubrig S., Järvinen S. P., Korhonen H., Ilyin I., Schöller M., Niemczura E., Chojnowski S. D., 2020, MNRAS, 495, L97

Hübscher J., 2011, Information Bulletin on Variable Stars, 5984, 1

Hümmerich S., Niemczura E., Walczak P., Paunzen E., Bernhard K., Murphy S. J., Drobek D., 2018, MNRAS, 474, 2467

Hümmerich S., Paunzen E., Bernhard K., 2020, A\&A, 640, A40

Hut P., 1981, A\&A, 99, 126

Kholopov P. N., Samus' N. N., Kazarovets E. V., Kireeva N. N., 1987, Information Bulletin on Variable Stars, 3058, 1

Kochukhov O., Adelman S. J., Gulliver A. F., Piskunov N., 2007, Nature Physics, 3, 526

Kochukhov O., et al., 2013, A\&A, 554, A61

Kochukhov O., Johnston C., Labadie-Bartz J., Shetye S., Ryabchikova T. A., Tkachenko A., Shultz M. E., 2021, MNRAS, 500, 2577

Korhonen H., et al., 2013, A\&A, 553, A27

Kukarkin B. V., Kholopov P. N., 1982, New catalogue of suspected variable stars

Luo A. L., Zhao Y. H., Zhao G., et al. 2018, VizieR Online Data Catalog, 5153,0

Maehara H., 2014, Journal of Space Science Informatics Japan, 3, 119

Maintz G., 2005, A\&A, 442, 381

Mathys G., 2004, in Maeder A., Eenens P., eds, IAU Symposium Vol. 215, Stellar Rotation. p. 270

Mennessier M. O., Colomé J., 2002, A\&A, 390, 173

Morel T., et al., 2014, A\&A, 561, A35

Netopil M., Paunzen E., Maitzen H. M., North P., Hubrig S., 2008, A\&A, 491,545

Nony T., et al., 2021, A\&A, 645, A94

North P., Debernardi Y., 2004, in Hilditch R. W., Hensberge H., Pavlovski K., eds, Astronomical Society of the Pacific Conference Series Vol. 318, Spectroscopically and Spatially Resolving the Components of the Close Binary Stars. pp 297-305

Otero S. A., Wils P., Hoogeveen G., Dubovsky P. A., 2006, Information Bulletin on Variable Stars, 5681, 1

Parenago P., 1946, Peremennye Zvezdy, 6, 45

Paunzen E., Wraight K. T., Fossati L., Netopil M., White G. J., Bewsher D., 2013, MNRAS, 429, 119

Paunzen E., Hümmerich S., Bernhard K., 2021, A\&A, 645, A34

Pojmanski G., 2002, Acta Astron., 52, 397

Preston G. W., 1974, ARA\&A, 12, 257

Pribulla T., et al., 2015, Astronomische Nachrichten, 336, 682

Pyatkes S. A., 1993, Bulletin of the Special Astrophysics Observatory, 35, 76

Ricker G. R., et al., 2014, Transiting Exoplanet Survey Satellite (TESS). p. 914320, doi:10.1117/12.2063489

Ricker G. R., et al., 2015, Journal of Astronomical Telescopes, Instruments, and Systems, 1, 014003

Samus N. N., Kazarovets E. V., Durlevich O. V., Kireeva N. N., Pastukhova E. N., 2017, Astronomy Reports, 61, 80

Schöller M., Correia S., Hubrig S., Ageorges N., 2010, A\&A, 522, A85

Smith K. C., 1996, Ap\&SS, 237, 77

Strassmeier K. G., Granzer T., Mallonn M., Weber M., Weingrill J., 2017, A\&A, 597, A55

Watson C. L., 2006, Society for Astronomical Sciences Annual Symposium, 25, 47

Wenger M., et al., 2000, A\&AS, 143, 9

White R. E., Vaughan A. H. J., Preston G. W., Swings J. P., 1976, ApJ, 204, 131

White T. R., et al., 2017, MNRAS, 471, 2882

Zeipel H., 1924, MNRAS, 84, 702

Zhao G., Zhao Y.-H., Chu Y.-Q., Jing Y.-P., Deng L.-C., 2012, Research in Astronomy and Astrophysics, 12, 723 


\section{Paunzen et al.}

This paper has been typeset from a $\mathrm{T}_{\mathrm{E}} \mathrm{X} / \mathrm{L}_{\mathrm{E}} \mathrm{X}$ file prepared by the author. 